\title{
Opiniones de tutores y residentes tras aplicar un sistema de evaluación formativa tipo portafolio: la nueva propuesta de 'Guía de práctica reflexiva' del Libro del especialista en Medicina de Familia en formación
}

\author{
R. Ruiz-Moral a , en representación del Grupo de Implementación del Portafolio del Residente \\ en la Unidad Docente de Medicina de Familia de Córdoba b
}

Introducción. La Comisión Nacional de la Especialidad de Medicina Familiar ha propuesto un nuevo Libro del especialista en Medicina de Familia en formación (LEF) tipo portafolio ('Guía de práctica reflexiva'). No existen en nuestro país experiencias que nos orienten sobre como este tipo de evaluación formativa puede aplicarse, desarrollarse y tampoco sobre su utilidad en la formación de residentes. Materiales y métodos. Estudio piloto cualitativo con encuestas estructuradas realizadas en una unidad docente provincial de Medicina de Familia. Durante nueve meses, 28 tutores ( $T$ ) y 36 residentes (R) participaron en una evaluación formativa de diferentes áreas competenciales, aportando tareas durante su trabajo. Los T y R respondieron a preguntas estructuradas y sus opiniones sobre contenidos, formato y posibles estrategias de aplicación fueron codificadas por dos evaluadores por temas recurrentes, explicaciones y conceptos. Resultados. Se recibieron encuestas de $33 \mathrm{R}$. Diecinueve T hicieron comentarios y 21 entregaron 67 informes de evaluación (media: 3 informes/tutor). Los Ty R consideran que el nuevo método puede ser útil para reflexionar sobre la práctica, conocer las competencias y fortalecer la relación tutor-residente, especialmente si se tienen en cuenta algunas sugerencias que mejorarían su aplicación y podrían reducir el tiempo y el esfuerzo empleado. Conclusiones. El nuevo LEF en su versión actual o con algunas modificaciones puede ser una herramienta para la evaluación formativa de los $\mathrm{R}$ de utilidad y probablemente bien aceptada en nuestro medio.

Palabras clave. Educación médica. Evaluación formativa. Portafolio. Residentes. Tutores de medicina de familia.
Trainers and trainees opinions after the pilot implementation of a formative evaluation: the new portfolio of the Spanish Family Medicine Resident

Introduction. The Spanish Family Medicine National Joint Commission is proposing a new trainee evaluation system type portfolio. In Spain, there is not previous experience that can guide about how this kind of formative assessment can be developed and we do not known about its usefulness in the trainees education. Materials and methods. Qualitative study with structured surveys carried out in a Provincial Family Medicine Teaching Unit. Along 9 months, 28 trainers and 36 trainees carried out a formative assessment on diverse competencies areas by means of specific tasks during their rotation in the Health Centres. Trainers and trainees filled an ad hoc survey and their comments on contents, format and possible implementation strategies were coded by two researchers in recurrent themes, explanations and concepts. Results. Thirty three surveys were received from trainees, 19 trainers made comments and 21 gave 67 evaluation reports (average: 3 by trainer). Both considered that the new method could be useful for reflecting on the clinical practice, for knowing better the own competencies and for strength the trainer-trainee relationship, especially if some suggestions related with its format and the way this is implemented are being in mind for the future. Conclusions. The new proposal of portfolio for family medicine trainees in this version or including some modifications can be well accepted and can be an useful tool for their formative evaluation.

Key words. Family medicine. Formative assessment. Medical education. Portfolio. Trainees. Trainers.

\footnotetext{
a Coordinador provincial.

b Tutores: M.J. Acosta,

C. Aguado, M. Amián,

F. Baena, J.M. Bueno,

J. Espinar, M.A. Fernández,

J.A. Fernández, L. García-

Vinuesa, S. Gascón,

J. Gavira, A. Hidalgo,

J. Martínez-De la Iglesia,

J. Merino, J. Mínguez, M.

Muñoz, A. Ortiz,

R. del Pozo, A. Roldán,

J. Ruiz-Moruno, P. Serrano,

F. Sierra-Duque, J. Solís,

A. Yun. Coordinadores

de área: J. González-Lama, C. Ortega-Millán.

Técnicos de Salud Pública:

M.J. Fernández-Fernández,

L. Pérula-De Torres, A. Valero.

Unidad Docente de

Medicina de Familia de

Córdoba. Córdoba, España.

Correspondencia Dr. Roger Ruiz Moral. Unidad Docente de Medicina de Familia de Córdoba. Blanco Soler, 4. E-14004 Córdoba.

E-mail

roger.ruiz.sspa@ juntadeandalucia.es

Agradecimientos A todos los residentes que han participado en esta supervisión. A los miembros de la CNMF, especialmente a su presidenta, V. Casado, por su empeño en el desarrollo de esta herramienta. A la Dra. M. Ezquerra, por compartir conmigo su trabajo y sus ideas en el portafolio del residente de Medicina de Familia.
} 


\section{Introducción}

Tras la elaboración por parte de la Comisión Nacional de Medicina de Familia (CNMF) de un nuevo Programa Oficial de la Especialidad (POE) en el año 2004 y su aprobación por este organismo y los ministerios de Sanidad y de Educación (BOE 3 de mayo de 2005) [1], desde la propia CNMF se planteó la necesidad de desarrollar un sistema de evaluación eficiente y acorde con los contenidos y la filosofía docente del nuevo POE. Así, bajo su auspicio, en marzo de 2004 se constituyó un grupo de trabajo compuesto por docentes ligados a diferentes unidades docentes de Medicina de Familia del país con el fin de elaborar un sistema de evaluación formativa, que culminó con una propuesta de un nuevo Libro del especialista en Medicina de Familia en formación (LEF), creada ya por la Orden de 22 de junio de 1995 [2] y cuya principal característica era la de incorporar una metodología de evaluación formativa tipo portafolio. Finalmente, esta propuesta se ha denominado 'Guía de práctica reflexiva' y se envió en abril de 2007 a través de la CNMF a todas las unidades docentes de Medicina de Familia del país para su aplicación como 'documento de recomendación'. Esta propuesta recoge los aspectos comunes de otras que definen la metodología portafolio en su aplicación formativa en ciencias de la salud [3-6] y que puede definirse como una recopilación de información y documentación en la que es posible encontrar pruebas, a través de la realización de una serie de tareas sugeridas, que reflejan que un proceso de aprendizaje ha sido realizado, así como del nivel alcanzado en los objetivos docentes previstos. En la medida en la que introduce la reflexión sobre la actuación, esta metodología aspira a representar un registro dinámico del crecimiento y cambio profesional [4]. Su finalidad es, pues, guiar la formación del residente hacia la competencia y madurez profesional. Todos estos elementos teóricamente favorecen el aprendizaje responsable del médico residente (autoaprendizaje) y contribuyen a la consolidación de la relación entre tutor y residente, pilar del proyecto educativo representado en el nuevo POE. De esta manera el modelo de portafolio presentado puede catalogarse como 'semiestructurado' o siguiendo la clasificación de Webb [7] del tipo 'Columna vertebral', ya que ofrece al residente una orientación sobre las com- petencias que debe dominar, en la mayoría de los casos indicaciones sobre el modo de valorarlas (indicadores operativos) y sugerencias sobre el material más idóneo que hay que aportar en cada caso (las tareas que se deben realizar). Esto marca diferencias con otros formatos de portafolios más libres [3], rígidos (estructurados) o con los 'libros de registro' o logbooks [8]. Así, sus principales consecuencias docentes deberían ser su capacidad formativa y evaluativa o el grado de personalización y autenticidad de los registros y reflexiones [5].

La enorme innovación que este tipo de herramienta docente-evaluativa supone sobre el actual sistema de evaluación de residentes imperante en nuestro país [9], unido a factores como el hecho de que algunas de sus principales virtudes educativas no han sido demostradas aún de forma directa y probablemente no lo sean nunca mediante la metodología del ensayo educativo (aunque tienen una gran coherencia lógica con las diferentes teorías de educación de adultos [10]), o el que hasta la fecha su uso en sus muy diversas y diferentes variantes se haya llevado a cabo principalmente en docencia pregraduada y, más recientemente, en la posgraduada y en sistemas de salud de ámbito anglosajón, justifica que cualquier propuesta en este sentido, y máxime si tiene un carácter oficial, deba ser tomada con precaución y adecuadamente supervisada teniendo en cuenta los condicionantes del nuevo ámbito [11]. Por ello, se diseñó un estudio cualitativo con tutores y residentes de una unidad de Medicina de Familia que supervisara los contenidos, el formato y las estrategias de aplicación posibles de este método de evaluación formativa tipo portafolio semiestructurado. En otra publicación hemos aportado y discutido otros aspectos de esta supervisión [11].

\section{Sujetos y métodos}

El formato de portafolio recomendado por la CNMF con el nombre final de 'Guía de práctica reflexiva' se caracteriza por ser un documento en soporte papel y electrónico, semiestructurado en función de las diferentes áreas competenciales del POE, y donde se priorizan competencias a trabajar a lo largo de la residencia, a la vez que se facilitan indicadores para la evaluación del grado 
Tabla I. Cuestionarios utilizados para recoger la información de residentes y tutores.

Cuestionario para los residentes

- ¿Qué ventajas crees que ha tenido trabajar con la metodología aportada por la guía-portafolio en comparación con la forma en la que hasta ahora hacías?

- ¿Cuáles son los inconvenientes?

- De cara al desarrollo futuro de esta herramienta docente, ¿qué sugerirías para que ésta pueda ser mejorada y sea más útil para el residente?

\section{Cuestionario para los tutores}

- ¿Cuáles han sido las ventajas principales que te ha supuesto desarrollar una tutorización a tu residente en esta área competencial con la metodología aportada por la guía-portafolio?

- ¿Cuáles son los inconvenientes?

- De cara al desarrollo futuro de esta herramienta docente, ¿qué sugerirías para que ésta pueda ser mejorada y sea más útil para la formación del residente?

en el que se alcanzan dichas competencias y se sugieren una gama de tareas como material de análisis y un modelo estructurado para realizar la reflexión en el que se invita al residente a detectar sus puntos fuertes y áreas de mejora, así como, en función de éstas, a planificar su formación. Todo este proceso debe de estar bajo la supervisión y el apoyo del tutor.

Se invitó a participar en la supervisión a un total de 28 tutores y 36 residentes ( 28 de tercer año y 8 de primero). Un cuestionario con tres preguntas abiertas sobre las ventajas, los inconvenientes y las sugerencias que el proceso en su globalidad, tanto para todas como para cada una de las competencias trabajadas con el residente, fue la base para recoger esta información de los tutores (Tabla I). A los primeros se les pidió también que valoraran las ventajas, los inconvenientes y las sugerencias que tras la experiencia tenían sobre el método y el proceso (Tabla I). La categorización de las respuestas tuvo en cuenta la bibliografía y los temas que emergieron de las propias opiniones de los tutores. El estudio tuvo lugar en un año. Inicialmente se llevaron a cabo talleres con tutores en los que se presentaron y discutieron los conceptos teóricos del aprendizaje de adultos y del modelo de portafolio propuesto, y se llevaron a cabo supuestos prácticos de aplicación en distintos escenarios clínicos y ante diferente material práctico (videograbaciones, incidentes críticos, sesiones clínicas, etc.). Igualmente se ensayaron formas de tutorización mediante el uso de portafolio que facilitaran su implementación al residente y para promover en él/ella un aprendizaje reflexivo. La información se analizó por dos investigadores mediante la codificación de temas recurrentes, explicaciones y conceptos que después cotejaron para su clasificación final. Los códigos se agruparon así en categorías en las que se incluyeron las opiniones diferentes. Se realizó una validación de los resultados obtenidos mediante la devolución de estos a todos los participantes en dos sesiones ad hoc.

\section{Resultados}

\section{Resultados de los residentes}

Se recibieron encuestas de un total de 33 residentes, de los que 25 eran de tercer año y 8 de primero. En la tabla II aparecen las competencias y las tareas trabajadas; entre las primeras destacan las cuatro promocionadas desde la unidad docente, especialmente comunicación, docencia y ética. Entre las tareas, la sesión clínica, el incidente crítico y la videograbación son las usadas con más frecuencia, y coinciden con el tipo de competencia trabajada.

\section{Opiniones de los residentes}

Para los residentes las ventajas principales de este método podían agruparse en:

- Favorecimiento del aprendizaje práctico: 'se da más importancia a la parte práctica y a la participación que a la teoría'; 'permite un aprendizaje basado en los problemas reales'; 'es muy útil para conocer los problemas'; 'permite un análisis profundo de situaciones que de otra manera pasaríamos por alto'.

- Mejora de la reflexión sobre los problemas clínicos: 'creo que tiene capacidad para que mejoremos no sólo en el motivo de la reflexión sino en el modo de realizarla en la práctica'; 'me ha sido de gran utilidad para observar mis errores y poder así mejorarlos'; 'integra la reflexión en la práctica habitual'. 
- Favorecimiento de un mayor conocimiento de las competencias del médico de familia: 'me ha ayudado a conocer y trabajar con las competencias de nuestra especialidad'; 'amplía tus alternativas de abordaje biopsicosocial.'

- Promoción de la relación con el tutor: 'nos ha obligado a tener una mayor interacción'; 'relación más activa entre el tutor y el residente, tanto por el papel que ha desempeñado como supervisor como por el feedback que me ha dado.'

Los inconvenientes descritos por los residentes estaban relacionados principalmente con:

- Las dificultades para escribir: 'a veces es muy complicado plasmar en el papel algunas incidencias'; 'no considero necesario plasmar por escrito las dudas, experiencias de la consulta clínica, ya que habitualmente las comentamos verbalmente y esto es totalmente válido, menos dificultoso y muy conveniente para la relación tutor-residente'.

- El proceso de reflexión exigido: 'la tarea de la autorreflexión tiene dificultad, por lo que muchas veces tiene de confidencial y... porque puede ser difícil ser crítico con uno mismo'.

- El tiempo que su realización exige: 'resta demasiado tiempo que se puede emplear en aspectos clínicos más útiles', y el problema que supone estar adecuadamente tutorizado: 'si no tienes un tutor colaborador te puedes sentir muy perdido'.

Lo que los residentes sugieren puede encuadrase dentro de las siguientes dos grandes categorías:

- Formación previa: 'necesitaríamos una formación y explicación del formato antes de realizarlo un poco más concreta para solucionar las dudas'; 'formar primero a los tutores para que nos orienten mejor'; 'es necesario que los tutores te orienten, te den la iniciativa para comenzar un portafolio.'

- Tiempo para adaptarse y experimentar con el método: 'creo que a lo largo de la residencia iré entendiendo qué es el portafolio y viendo si me ha sido útil o no'; 'me parece interesante, pero aún sin la suficiente experiencia previa no se que repercusión puede tener en mi formación'; 'supongo que cuando esté totalmente establecido las normas estarán más claras y nos ayudará a tener más relación entre tutor y residente'.

\section{Resultados de los tutores}

Participaron 28 tutores, de los que 19 hicieron cometarios cualitativos y 21 entregaron un total de 67 informes de evaluación de los informes de reflexión sobre la competencia y las tareas realizadas por su/s residente/s (media: 3 informes/tutor) (Tabla II). Los resultados sobre el área competencial trabajada y la tarea suministrada, así como su calidad, se aportan en la otra parte de este trabajo antes referida. Entre las ventajas se destacan aspectos relacionados con:

- La necesidad de disponer de espacios y tiempos específicos para la formación: 'exige buscar un tiempo concreto para dedicarlo a la tutorización, se formaliza una actividad cotidiana'.

- El incremento de las responsabilidades docentes para los tutores que esto les supone: 'hace que como tutor te pongas las pilas, te recicles, percibas tus propias carencias y busques respuestas para luego poder dárselas al residente.

- Pero igualmente se resaltaba el papel orientativo del formato semiestructurado: 'tener yo claro lo que me falta a mi por aprender y hacerlo y lo que le falta a él', 'hace explícitos los objetivos y las tareas del aprendizaje.

- Una gran cantidad de tutores coincidía en que: 'mejora la relación tutor-residente, ya que se reflexiona juntos, se comentan sensaciones, etc..'

- La obligatoriedad de registrar fue objeto de muchos comentarios: 'el papel obliga a concretar, ordenar y sintetizar ésta es su ventaja y su inconveniente. Es como una foto sobre la que se intenta leer, en voz alta, su contenido: se da una sistemática que guíe esa lectura y así, con detenimiento, menos cosas se nos quedarán en el tintero', 'el residente, así, se obliga a profundizar y actualizar sus conocimientos y plasmarlos por escrito', 'y se esfuerza más', 'mayor profundidad y eficacia en el análisis'.

- La experiencia les hace resaltar a los tutores muy frecuentemente la bondad del método sobre la reflexión: 'exige pararse a pensar en aquellas situaciones que han sido consideradas como problema u oportunidad de aprendizaje,' 'la reflexión hace que me verbalice du- 
Tabla II. Competencias y tareas trabajadas por los residentes con metodología portafolio.

\begin{tabular}{|c|c|}
\hline \multicolumn{2}{|l|}{ Area competencial } \\
\hline Comunicación & $59 \%$ \\
\hline Docencia & $58 \%$ \\
\hline Ética & $51 \%$ \\
\hline Atención a la familia & $39 \%$ \\
\hline Atención al individuo & $33 \%$ \\
\hline Razonamiento clínico & $24 \%$ \\
\hline Atención a la comunidad & $21 \%$ \\
\hline Investigación & $21 \%$ \\
\hline Atención a grupos especiales & $12 \%$ \\
\hline Gestión clínica & $3 \%$ \\
\hline \multicolumn{2}{|l|}{ Tarea } \\
\hline Incidente crítico & $61 \%$ \\
\hline Sesión clínica & $51 \%$ \\
\hline Vídeograbación-consulta & $48 \%$ \\
\hline Caso clínico & $36 \%$ \\
\hline Proyecto de investigación & $24 \%$ \\
\hline Informe & $12 \%$ \\
\hline Revisión de historia clínica & $9 \%$ \\
\hline Trabajo de campo & $6 \%$ \\
\hline Otros & $15 \%$ \\
\hline
\end{tabular}

das y logros. A veces tanto unos como otros quedan poco evidentes para el tutor y sobreentendemos conocimientos o los infravaloramos', 'la actividad de autorreflexión resulta más estructurada'. Sobre el autoaprendizaje: 'ayuda al residente a descubrir sus lagunas y limitaciones, y así orientar mejor sus esfuerzos'; 'da protagonismo al residente'; 'aumenta la capacidad para detectar autodeficiencias formativas'.
- Sobre el aprendizaje de adultos-experiencia: 'se basa en hechos reales y por lo tanto en situaciones que se pueden encontrar en su práctica clínica futura'; 'se desarrolla a partir de la propia experiencia'; 'se trabaja sobre necesidades reales del residente.

- Sobre la evaluación: 'ofrece un instrumento de evaluación más objetivo'.

En cuanto a los inconvenientes:

- El tiempo es el que merece un mayor número de comentarios junto a la carga de trabajo adicional que supone: 'el análisis puede precisar hasta dos horas o más para realizarlo'; 'requiere un tiempo que no siempre tenemos o podemos encontrar'; 'la falta de tiempo. Nuestras consultas están saturadas y los residentes también tienen otras muchas actividades (cursos, máster, etc.)'; 'escribir, poner nombre a las cosas, ordenarlas, recapacitar y seguir poniendo nombre a las cosas, intercambiar enseñanzas y propósitos que naturalmente deben llevarse a cabo es un proceso muy laborioso'; 'no me parece coste-eficiente: requiere mucha dedicación y no creo que mejore el sistema de evaluación basado en la observación directa'.

- Hay comentarios relacionados con el rechazo a lo nuevo y a la forma de introducirlo: "la imposición crea resistencias y eso bloquea el aprendizaje'; 'metodología nueva: genera ansiedad y resistencia al cambio'; 'el principal problema es la falta de costumbre de uso de esta metodología. La rutina diaria y la resistencia al cambio de pautas habituales de comportamiento.

- También son frecuentes los comentarios sobre la necesidad de una formación específica en el método: 'necesita un período de adaptación a la nueva mecánica de aprendizaje, en otras palabras, hace falta estudiar más, formarse más, es como si a un profesor le cambian el temario de un año para otro y se le pide que dé más y mejor de lo que ya está dando'; 'dificultades inherentes a la utilización de una nueva herramienta: aprendizaje, familiarización, comprensión.

- La dificultad que tienen algunos para escribir fue objeto de críticas similares que pueden ejemplificarse en: 'dificultad para transcribir al lenguaje escrito nuestras reflexiones'.

- Su validez como método también es objeto de reflexión: 'es lo que se estaba haciendo, pero 
ahora hay que dejarlo por escrito, ¿merece realmente la pena?'

Finalmente, lo que los tutores sugerían podía agruparse como:

- Aportaciones sobre el formato: 'la cosa puede ser más liviana si se hace en forma de notas o pequeños apuntes'; 'mejor ahondar en su calidad y estilo, ¿dos tipos de portafolio, el tipo apunte/notas y/o la novela narrativa?'; 'simplificar guías'; 'es necesario simplificar el proceso: se tarda casi una hora y media'.

- Sobre estrategias generales para su mejor implementación o aprovechamiento, relacionadas sobre todo con la organización para la optimización del tiempo dedicado: 'establecer modificaciones de horarios para crear espacios de discusión'; 'utilizar el correo electrónico'; 'hemos habilitado ya en el centro espacios concretos para trabajarlo, por ejemplo, dos días al mes de formación continuada se dejan libres para esto; 'otras veces, de los días liberados de consulta a los residentes alguno será para ello'. Con estrategias educativas: 'creo que el aprendizaje necesita cierta inmediatez o tener un soporte grabado para que sea eficaz'; 'se debe partir siempre de necesidad sentida'; 'trabajar de forma conjunta y continuada, desde el primer día con esta herramienta. Evitar el trabajo mediante 'arreones'; 'que el residente tenga claro desde el primer día qué es lo que se espera que haga y cómo lo puede conseguir de cara a su formación.

- Con aspectos relacionados con los incentivos: 'que la empresa valore y premie el portafolio del residente: aparte de aprobar o pasar la residencia globalmente, que a los residentes con los mejores portafolios se les premie con los mejores puestos de trabajo (vacantes, sustituciones, etc.)'.

- Igualmente, los tutores insistieron sobre la necesidad de formación: 'la enseñanza de calidad requiere una preparación adecuada que el tutor debe recibir'; 'plantear talleres o cursos previos a los tutores y residentes sobre la dinámica del porfolio'.

- Finalmente, una serie de aspectos que preocupan y deberían clarificarse: 'clarificar si nos dirigimos hacia un portafolio formativo, evaluativo o mixto'; ‘ ¿este sistema es mejor que el previo o sólo aporta una mayor estructuración?'; 'antes de implantarlo: supervisarlo'.

\section{Conclusiones}

Si se atiende tanto al número de residentes y tutores que han participado en esta supervisión como al número y variedad de competencias trabajadas y tareas presentadas, la presente supervisión se puede considerar adecuada para sus fines, y sus resultados válidos para ser extrapolables a otros ámbitos similares y para ser inspiración de modificaciones futuras en el LEF, motivo éste principal de su puesta en práctica. Aunque se ha comunicado una experiencia previa de utilización experimental de una herramienta tipo portafolio, ésta ha sido realizada con un menor número de residentes y tutores de Medicina de Familia y con un formato de portafolio diferente y más simple (dirigido únicamente al análisis del tratamiento de pacientes crónicos) que el aquí utilizado [12]. De forma global podemos decir que la propuesta ensayada ha sido bastante bien recibida y calificada tanto por los residentes, a los que va principalmente dirigida, como por sus tutores. Para conocer los comentarios específicos que generan estos resultados deberíamos tener presente los objetivos principales que desde la CNMF se pretenden alcanzar con la instauración de este LEF y que no son otros que los de disponer de una metodología que sea aplicable en las circunstancias reales en las que se desarrolla la formación de los residentes y que les sirva para su evaluación formativa, que promueva, sobre todo la reflexión, sobre las actuaciones prácticas que le son propias a la vez que, con estos fines, se fortalezca la relación tutorial. Respecto al papel del portafolio para un aprendizaje reflexivo sobre las actuaciones propias de su práctica, la impresión generalizada de ambos actores es que efectivamente mejora el proceso de reflexión. Son muchos los comentarios por las dos partes en este sentido. Basándose sobre todo en la idoneidad de este método y las teorías educativas de adultos [13], pero también en las impresiones de otros que los han utilizado, ésta sería una de las misiones más importantes del portafolio $[5,4,14,15]$. Sin embargo, para que esto suceda, distintos estudios muestran que se deben dar una serie de condicionantes, que en gran medida los participantes en esta experiencia han intuido. Así, la importancia de la relación tutorresidente, que los tutores estén adecuadamente entrenados [6,15-19], o la necesidad de planificar cuidadosamente su introducción $[6,19]$ merecen amplios comentarios por parte de ambos. En lo 
que respecta a esto último, Challis [6] señala que la aceptabilidad de una metodología de este tipo puede depender de ello. En este sentido nuestros residentes y tutores mostraron preocupación por este tema, en gran medida motivado por el hecho de que especialmente muchos de los primeros vivieron este proceso como una preocupación más de la Unidad Docente que como algo de interés para ellos. Para nuestros tutores y residentes esta planificación debería incluir una definición previa de los objetivos que se pretenden alcanzar y del papel que se espera desempeñe cada uno, algo también señalado en otros estudios $[15,18,20]$.. La aceptabilidad dependería también del formato físico y de que éste posea una estructura clara y amigable $[6,19,20]$, igualmente reflejado ampliamente en los comentarios de nuestra muestra. De esta manera, una introducción a gran escala, poco cuidada y con un formato poco adaptado podría ser un fiasco incluso con supervisión. En este sentido nuestros médicos proponen simplificar al máximo, algo difícil de realizar si se mantiene el formato semiestructurado. Sin embargo, al igual que los tutores y residentes de los estudios de Dundee [16,17], los nuestros reconocen que el formato actual tiene la ventaja de orientar no sólo sobre el modo de usarse y los pasos que se deben seguir, sino sobre el tipo de competencias y habilidades que se espera que un médico de familia desarrolle. Tal vez el formato para la autorreflexión sea susceptible de una mayor simplificación; con este fin se puede dejar el esfuerzo de escribir sólo para el resumen, los aspectos positivos, los mejorables y la forma de adquirirlos y potenciar todos los demás para la discusión entre tutor y residente. Modificaciones en este sentido pueden ser importantes ya que las habilidades para escribir no suelen ser buenas en todos los residentes y además se ha demostrado una correlación positiva entre estas habilidades y una valoración positiva del portafolio [21]. La orientación que favorece el portafolio hacia una formación eminentemente práctica es resaltada por ambos grupos, lo que interpretamos como un hecho significativo que destaca esta modalidad de aprendizaje frente a otras frecuentemente ofrecidas en nuestro medio. También otros estudios con poblaciones similares resaltan estas facetas $[12,16,17]$. Efectivamente, las características de esta metodología la situarían en el vértice de la pirámide de Miller [22] y se enmarca perfectamente en el concepto de aprendizaje reflexivo y basado en los problemas [10].
Un aspecto importante señalado en ambos grupos es el de la necesidad ahora de disponer de espacios de tutorización específicos y previamente acordados. Esto es especialmente importante en nuestro ámbito donde sólo una de cada 10 parejas docentes planificaban estos espacios antes de la introducción del portafolio. Esta necesidad es observada por muchos como algo positivo, ineludible y que habría que integrar en el trabajo diario, lo que se presenta como una buena oportunidad para gestionar mejor el tiempo de un médico-docente y para, principalmente de cara a la administración, marcar diferencias con otros profesionales o centros sin responsabilidades de este tipo. Esto, sin embargo, no quita que el portafolio sea visto como una sobrecarga importante que, a falta de pruebas de eficacia evidentes frente a otros métodos, levante reticencias. Éstas son mayores en lo referente al tiempo que conlleva su realización, el cual, aunque es probable que sea menor una vez se tenga más experiencia, también ha sido señalado por otros estudios como una razón contra su uso [17,23].

Un aspecto relacionado con la consideración, generalizada que antes comentábamos de fomentar la reflexión es la validez o profundidad de esta, que parece perderse a medida que transcurre más tiempo entre la reflexión y la experiencia objeto de la misma [19], algo por otro lado lógico. Es, por tanto, deseable que la reflexión escrita se realice inmediatamente tras la experiencia docente, lo que quizás sea favorecido por la simplificación del formato del informe de autorreflexión. Otro aspecto que algún estudio [18] ha resaltado como importante a la hora de valorar la utilidad de un portafolio es que los que lo usen dispongan de una variedad de experiencias suficiente, algo que tal vez pueda representar un problema en el grado pero que no debería de serlo con residentes, salvo que éstos no tengan el papel activo en la asistencia a pacientes que deben tener en cada momento. Los tutores deberían tener esto en cuenta y fomentar la actividad del residente adecuadamente tutorizada. En nuestro estudio la media de actividades realizadas fue de tres por pareja; así, es probable que con este número muchos residentes y tutores no haya captado las autenticas potencialidades y limitaciones del método.

Por otra parte, los residentes fueron casi unánimes en considerar que este método no debe ser utilizado para la evaluación sumativa. Es cierto que las auténticas potencialidades formativas del 
portafolio pueden verse seriamente alteradas al transformarlo en una herramienta de evaluación para la certificación. La estandarización que ello requiere con el objeto de obtener índices de fiabilidad aceptables disminuye su autenticidad y personalización $[5,14]$, a la vez que induce sesgos conscientes en los informes de reflexión y en la elección del material $[4,14]$. Sin embargo, y paradójicamente, se ha visto que los estudiantes son menos propensos a usarlos si no hay una evaluación [24]. Snadden [17], en un estudio similar al nuestro, observó que cuando llegaba la época de exámenes los Residentes dejaban de hacerlo y Driessen tras un estudio con tutores de pregrado aboga por su uso sumativo para conseguir que el método sea tomado en serio [18]. Creemos que este debate no tiene ahora su momento, pero en la medida en que progresivamente se instauren evaluaciones objetivas de las competencias para certificar la especialidad, el portafolio debería ser contemplado como un método evaluativo más cuyos pros y contras habría sin duda que considerar.

Para concluir, existe una preocupación especialmente manifestada por nuestros tutores, algún otro docente [25] y ciertos autores [19] acerca de la eficiencia del portafolio, sobre todo, y como hemos señalado, si se tiene en cuenta su coste. Aunque ya hemos apuntado que las impresiones de la mayoría de los que lo han usado son muy positivas y encaja perfectamente en la lógica de la educación de adultos y en el tipo de metodología que favorece el aprendizaje significativo y modificador de conductas [26], es verdad que aún las evidencias en este sentido son indirectas y habría que diseñar estudios que, en lo que respecta a la promoción de la reflexión, demuestren con más claridad el valor que el portafolio añade a otros sistemas de tutorización más o menos imperantes entre nosotros, como, la simple supervisión por parte del tutor, el análisis significativo de acontecimientos o el análisis de casos. Mientras esto llega, no existen razones de peso que impidan su puesta en práctica.

Finalmente, diremos que esta supervisión parece poner de manifiesto que la nueva 'Guía de práctica reflexiva'-LEF en su versión actual o con algunas modificaciones puede ser una herramienta para la evaluación formativa de los residentes de utilidad y probablemente bien aceptada, especialmente si se tienen en cuenta algunas recomendaciones fruto de esta supervisión y que, a modo de conclusiones, exponemos a continuación:
- La introducción de la 'Guía de práctica reflexiva' en cada una de las unidades docentes debe estar cuidadosamente planificada.

- Esto exigirá una formación previa de tutores en su manejo y en el de otras técnicas de educación de adultos.

- Los residentes deben conocer perfectamente lo que se espera que hagan con esta metodología y los responsables docentes deberían garantizar una tutorización adecuada durante todo el proceso.

- En el momento presente, el formato presentado puede ser suficientemente claro y facilitador, pero es susceptible de incorporar mejoras en este sentido, que muy probablemente deban ser valoradas de forma local.

- Tal vez cada unidad docente o pareja tutorresidente debería tomarlo inicialmente como un modelo modificable en sus detalles a medida que sea utilizado.

- El formato semiestructurado parece adecuado para orientar a tutores y residentes sobre las áreas competenciales y habilidades que deben trabajar.

- Las tareas propuestas en el formato actual representan sugerencias y cualquier unidad docente o pareja tutor-residente que conozca bien la competencia que se debe trabajar podría proponer nuevas tareas para su demostración.

- Su puesta en práctica exige al tutor y residente la planificación de espacios de tutorización específicos en sus actividades habituales. Esta planificación puede ser también planificada desde la organización del centro.

- Las tareas de la guía-portafolio pueden ser trabajadas en gran medida de manera virtual.

- La reflexión que el residente realice sobre cualquier competencia o conjunto de habilidades trabajadas debería completarse casi inmediatamente después del análisis de la tarea y no dejar pasar demasiado tiempo.

- El desarrollo por parte del tutor de este tipo de tareas docentes debe ser reconocido por la administración sanitaria, tanto en lo que respecta a la organización de su trabajo diario como en los incentivos contemplados en cada administración para la carrera profesional y docente.

- Debe quedar claro que se trata de una evaluación formativa de la que el residente es el responsable y que sus reflexiones no van a ser objeto de análisis y para fines sumativos. 
A modo de conclusión general, una recomendación podría ser que tanto los tutores como los residentes deben ver en esta herramienta una forma de ayuda para reflexionar sobre la competencia profesional y que el objetivo, por lo tanto, no es 'rellenar los papeles del portafolio' sino aprovechar el mayor número de experiencias prácticas significativas para profundizar y avanzar en el crecimiento profesional mediante la reflexión guiada.

\section{Bibliografía}

1. Ministerio de Sanidad y Consumo. Programa Formativo de la Especialidad de Medicina Familiar y Comunitaria. Madrid: Ministerio de Sanidad y Consumo; 2004

2. Comisión Nacional de Medicina de Familia. Libro del especialista en Medicina de Familia y Comunitaria en formación: Guía de portafolio. Ezquerra M, Ruiz-Moral R, eds. Madrid: Comisión Nacional de la Especialidad de Medicina de Familia. Ministerios de Sanidad y Consumo y Educación y Ciencia; 2006.

3. Working Group on Higher Professional Education. Portfolio-based learning in General Practice. London: Royal College of General Practitioners; 1993. Report No.: Occasional papper 63.

4. McMullan M, Endacott R, Gray M, Jasper M, Miller C, Scholes J, et al. Portfolios and assessment of competence: a review of the literature. J Advan Nursing 2003; 41: 283-94.

5. Friedman B, Davis M, Harden R, Howie P, Ker J, Pippard M. AMEE Medical Education Guide No. 24: Portfolios as a method of student assessment. Medical Teacher 2001; 23: 535-51.

6. Challis M. AMEE Medical Education guide No. 11 (revised): Portfolio-based learning and assessment in medical education. Medical Teacher 1999; 21: 370-86.

7. Webb C, Endacott R, Gray M, Jasper M, Miller C, McMullan M, et al. Models of portfolios. Med Educ 2002; 36: 897-8.

8. University of Leeds. URL: http://www.bradfordvts.co.uk/ onlineresources/downloadableforms/GPR\%20Logbook. doc. [02.11.2006].

9. Ministerio de la Presidencia. Orden de 22 de junio de 1995 por la que se regulan las Comisiones de Docencia y los sistemas de evaluación de la formación de los médicos y de farmacéuticos especialistas. BOE 1995, 30 de junio de 1995: 19793-9.

10. Novak J. Conocimiento y aprendizaje. Madrid: Alianza Editorial; 1998.
11. Ruiz-Moral R, Grupo de implementación del portafolio del residente en la Unidad Docente de Medicina de Familia de Córdoba. Estudio piloto para la incorporación del portafolio del Libro del especialista en Medicina de Familia en formación. Aten Primaria 2007; 39: 479-82.

12. Torán-Montserrat P, Arnau-Figueras J. El portafolio como instrumento de valoración del residente. Aten Primaria 2006; 37: 371-3.

13. Spencer J, Jordan R. Learner centred approaches in medical education. BMJ 1999; 318: 1280-3.

14. Roberts C, Newble D, O'Rourke A. Portfolio-based assessments in medical education: are they valid and reliable for sumative purposes? Med Educ 2002; 36: 899-900.

15. Driessen E, Van Tartwijk J, Vermunt J, Van der Vleuten C. Use of portfolios in early undergraduate medical training. Med Teach 2003; 25: 14-9.

16. Snadden D, Thomas M, Griffin E, Hudson H. Portfolio based learning in general practice vocational training. Med Educ 1996; 30: 148-52.

17. Snadden D, Thomas M. Portfolio learning in general practice vocational training -does it work? Med Educ 1998; 32: 401-6.

18. Driessen E, Van Tartwijk J, Overeem K, Vermunt J, Van der Vleuten C. Conditions for successful reflective use of portfolios in undergraduate medical education. Med Educ 2005; 39: 1230-35.

19. Pearson D, Heywood P. Portfolio use in general practice vocational training: a survey of GP registrars. Med Educ 2004; 38: 87-95.

20. Wade R, Yarbrough D. Portafolios: a tool for reflective thinking in teacher education? Teach Teacher Educ 1996; 12: 63-79.

21. Mitchell M. The view of students and teachers on the use of portfolios as a learning and assessment tool in midwifery education. Nurse Education Today 1994; 14: 38-43.

22. Miller G. The assessment of clinical skills/competence/ performance. Acad Med 1990; 65: S63-7.

23. French F, Valentine M. An evaluation of the introduction of personal learning plans for general practitioners in Grampian region (north-east Scotland). Educ Gen Pract 2000; 11: 165-74.

24. Harris S, Dolan G, Fairbairn G. Reflecting on the use of student portfolios. Nurse Education Today 2001; 21: 278-86.

25. Morera Montes J. Reflexiones sobre la factibilidad y aplicabilidad del portfolio. Aten Prim 2006; 37: 243.

26. Davis D, Thomson O'Brien M, Freemantale N, Wolf F, Mazmanian P, Taylor-Vaisey A. Impact of formal continuing medical education. Do conferences, workshops, rounds, and other traditional continuing education activities change physician behavior or health care outcomes? JAMA 1999; 282: 867-74. 